\title{
Automatic Alignment of Renal DCE-MRI Image Series for Improvement of Quantitative Tracer Kinetic Studies
}

\author{
Darko Zikic $^{1}$, Steven Sourbron ${ }^{2}$, Xinxing Feng ${ }^{1}$, Henrik J. Michaely ${ }^{2}$, \\ Ali Khamene ${ }^{3}$, and Nassir Navab ${ }^{1}$ \\ ${ }^{1}$ Computer Aided Medical Procedures (CAMP), Technische Universität München, Germany \\ 2 Institute of Clinical Radiology, University Hospitals Munich-Grosshadern, Germany \\ 3 Siemens Corporate Research (SCR), Princeton, USA
}

\begin{abstract}
Tracer kinetic modeling with dynamic contrast enhanced MRI (DCE-MRI) and the quantification of the kinetic parameters are active fields of research which have the potential to improve the measurement of renal function. However, the strong coronal motion of the kidney in the time series inhibits an accurate assessment of the kinetic parameters. Automatic motion correction is challenging due to the large movement of the kidney and the strong intensity changes caused by the injected bolus. In this work, we improve the quantification results by a template matching motion correction method using a gradient-based similarity measure. Thus, a tedious manual motion correction is replaced by an automatic procedure. The only remaining user interaction is reduced to a selection of a reference slice and a coarse manual segmentation of the kidney in this slice. These steps do not present an overhead to the interaction needed for the assessment of the kinetic parameters. In order to achieve reliable and fast results, we constrain the degrees of freedom for the correction method as far as possible. Furthermore, we compare our method to deformable registration using the same similarity measure. In all our tests, the presented template matching correction was superior to the deformable approach in terms of reliability, leading to more accurate parameter quantification. The evaluation on 10 patient data series with 180-230 images each demonstrate that the quantitative analysis by a two-compartment model can be improved by our method.
\end{abstract}

Keywords: Registration, DCE-MRI

\section{NEW WORK TO BE PRESENTED}

We present our work on improvement of the results of quantitative tracer kinetic studies in renal DCE-MRI, by applying a contrast-invariant motion correction scheme, based on template-matching. The comparison to the deformable method using the same similarity criterion suggests that for this application, approaches with low number of degrees of freedom can be superior because of the inherent reliability.

\section{INTRODUCTION}

Dynamic contrast enhanced MRI (DCE-MRI) is a method that visualizes the kinetics of body fluids by rapid injection of a bolus of contrast agent, and dynamic imaging of the bolus passage in an organ of interest. Examples images of DCE-MRI of the kidney are shown in Figure 1. In recent years, the use of DCE-MRI for the characterization of renal function has received increasing attention in the literature. ${ }^{1}$ In principle, the method allows for a quantitative, local measurement of two complementary markers of renal function: perfusion and glomerular filtration. Moreover, DCE-MRI can be combined with anatomical imaging, angiography and functional methods such as oxygenation- and diffusion weighted imaging to provide a complete non-invasive assessment of renal status in a single exam. ${ }^{2}$

Further author information: (Send correspondence to Darko Zikic)

Darko Zikic: E-mail: zikic@in.tum.de

Steven Sourbron: E-mail: steven.sourbron@med.uni-muenchen.de

Xinxing Feng: $\quad$ E-mail: fengxi@in.tum.de

Henrik J. Michaely: E-mail: henrik.michaely@med.uni-muenchen.de

Ali Khamene: E-mail: ali.khamene@siemens.com

Nassir Navab: $\quad$ E-mail: navab@in.tum.de 


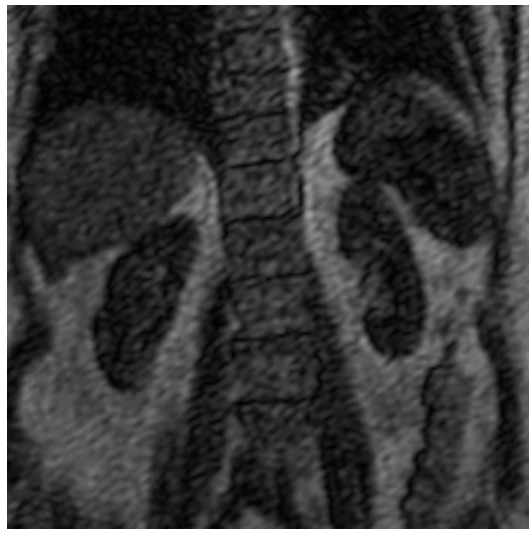

(a) Image 2

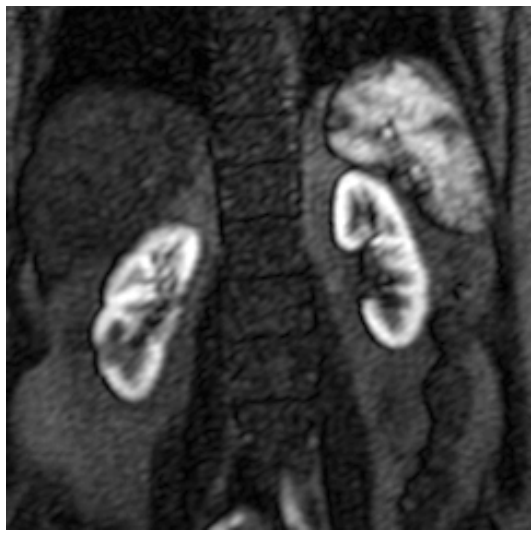

(b) Image 27

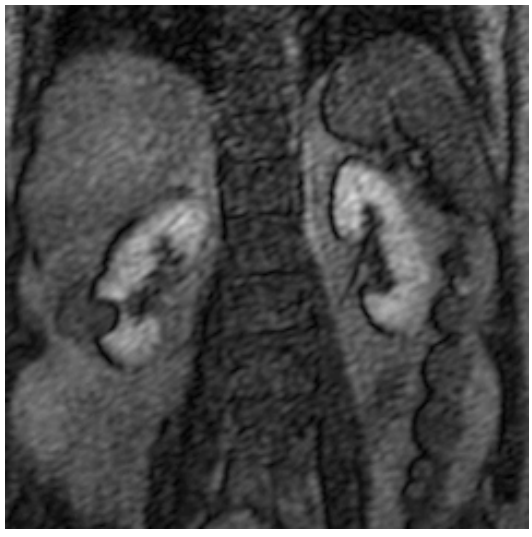

(c) Image 177

Figure 1: Three exemplary images from a DCE-MRI sequence demonstrate the strong intensity changes and apparent geometry changes in the images.

Tracer kinetic modeling with DCE-MRI and the quantitative evaluation of the kinetic parameters is a particularly active field of research, because the superior spatio-temporal resolution of MRI offers possibilities that are not available to nuclear medicine renography, the current gold-standard method for measuring renal function. Quantification of functional kinetic parameters with DCE-MRI requires the measurement of two separate time-courses: one in the tissue of interest (which can be a pixel or larger region), and one in a large artery feeding the tissue. The extraction of physiologically relevant information from these data consists of two steps. First, the concentration-time courses of contrast agent are calculated from the dynamic MRI signals. Second, the principles of tracer kinetic theory ${ }^{3}$ are applied to derive the tissue characteristic parameters from a comparison between arterial- and tissue concentrations.

In the kidney the arterial measurement is performed in the aorta, which is stable in the axial slice during breathing motion. However the kidneys move during breathing in the coronal slices, so some form of motion compensation must be applied in order to derive quantifiable time courses. One option is to perform the measurement during breath hold, but this is not feasible for a measurement of filtration where an acquisition time of several minutes is required. Another possibility is respiratory gating, but this reduces the temporal resolution to values that are too low for a perfusion measurement. An ideal solution would be to perform the measurement at high temporal resolution during free breathing, and correct for the motion on the level of post-processing by registration.

To this end, we employ a template-matching based motion correction scheme solving for translational parameters only. The results demonstrate that this method provides a clear improvement of the results for the application of quantitative parameter analysis. Our tests also indicate that this approach is better suited for the application than deformable methods with a larger number of degrees of freedom (DOF) using the same similarity criterion, since it preserves the shape of the kidney. The large intensity variations which are present in the perfusion scans can lead the deformable methods to change the shape of the kidney, which results in errors in the estimation of the kinetic parameters. Besides, the translation correction model also leads to significantly lower runtimes.

Different previous work on registration of MR perfusion images as well as some related work is reported in the literature. Bansal and Funka-Lea ${ }^{4}$ use a template matching method, which recovers the translation parameters using mutual information (MI), for cardiac data. Sun et al. ${ }^{5}$ present a framework for affine registration of MR perfusion images, tested on cardiac and renal data. The approach uses a gradient-based similarity criterion, similar to the one employed in this paper. El-Baz et al. ${ }^{6}$ present a CAD system for evaluation of kidney diseases using DCE-MRI. Here the main objective is the automatic classification of the dynamics*. To this end, segmentation and deformable registration of kidney structures are employed.

\footnotetext{
*A dynamic is the intensity evolution at one position in the image over time.
} 


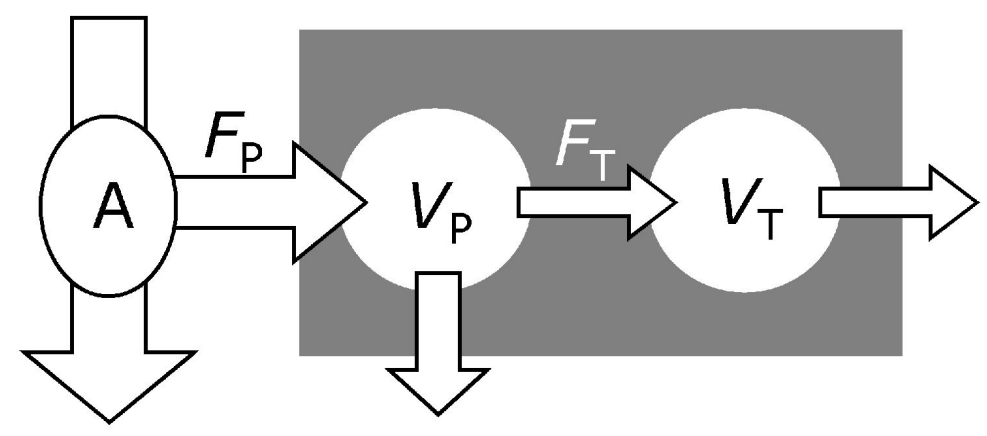

Figure 2: Diagram of the two-compartment model for the kidney or kidney cortex. The plasma flow $\left(F_{\mathrm{P}}\right)$ carries the contrast agent from an arterial region (A) into the tissue (grey rectangle). It first enters the tissue plasma, where it distributes over the plasma volume $\left(V_{\mathrm{P}}\right)$. A fraction of the entering contrast agent is filtered out of the vascular compartment and is carried by the tubular flow $\left(F_{\mathrm{T}}\right)$ into the tubular system where it distributes over the tubular volume $\left(V_{\mathrm{T}}\right)$. The contrast agent leaves the tissue carried by the outflow out of the vascular and tubular compartments.

\section{METHODS}

In the following, we present the methods involved in the improved quantitative tracer kinematic approach. First, we describe the data acquisition setup. We go on by explaining the motion correction scheme. Finally, we briefly describe the employed tracer kinematic modeling and the actual workflow for the estimation of the the perfusion and filtration parameters.

\subsection{Data Acquisition Setup}

During the DCE-MRI scan a series of images is acquired. Here, four slices ( 3 coronal, 1 axial) are acquired in an alternating manner, such that the temporal resolution between the respective slices is approximately 1 second. For our method, we assume that the main motion of the kidney takes place in the coronal slices. The analysis of the kidney motion in the axial slice confirms this assumption. During the acquisition there exists an observable drift of the mean of breathing motion. Therefore, the acquisition protocol was changed from breath hold at beginning to normal breathing during the whole scan. Although this step in general already "normalizes" the breathing motion, for some patients a change in the breathing motion is still present.

\subsection{Alignment of Image Series by Template Matching}

The two main challenges for registration of the large number images in perfusion scans are the following: first, extremely strong intensity variations over the time series, which can lead to apparent geometry changes, and second, large displacements between single images in the time series.

For the assessment of kinetic parameters, the shape of the kidney structures has to be maintained during the motion correction process. Since the perfusion studies exhibit a high rate of intensity variation over time, this constraint is hard to enforce with a deformable model. On the other hand, the rigid motion model implicitly maintains the structures during registration, thus adding robustness to the process. Since the major motion of the kidney can be captured by a translation and the computation time is important for the application, we chose to constrain the rigid motion to translational movement only. Therefore, we employ a template matching technique.

As similarity measure, we use a gradient-based measure discussed for example by Haber and Modersitzki, ${ }^{7}$ where it is referred to as normalized gradient field (NGF). For two images $I_{1}$ and $I_{2}$ with $N$ points, the NGF similarity measure can be defined as

$$
S_{\mathrm{NGF}}\left(I_{1}, I_{2}\right)=\frac{1}{2 N} \sum_{i=1}^{N}\left\langle\mathbf{n}_{\epsilon}\left(I_{1}, \mathbf{x}_{i}\right), \mathbf{n}_{\epsilon}\left(I_{2}, \mathbf{x}_{i}\right)\right\rangle^{2}
$$




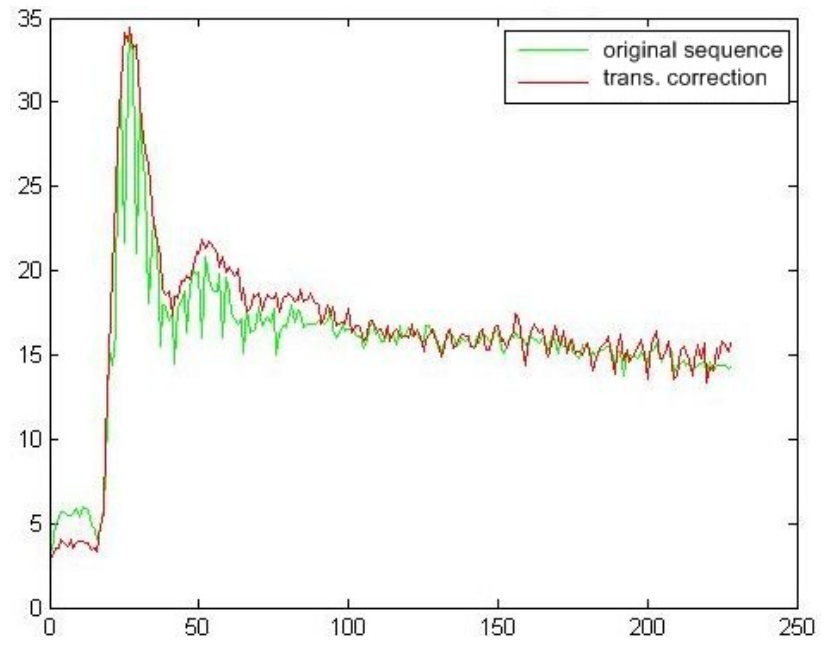

(a) Dynamics (Perfusion Curves)

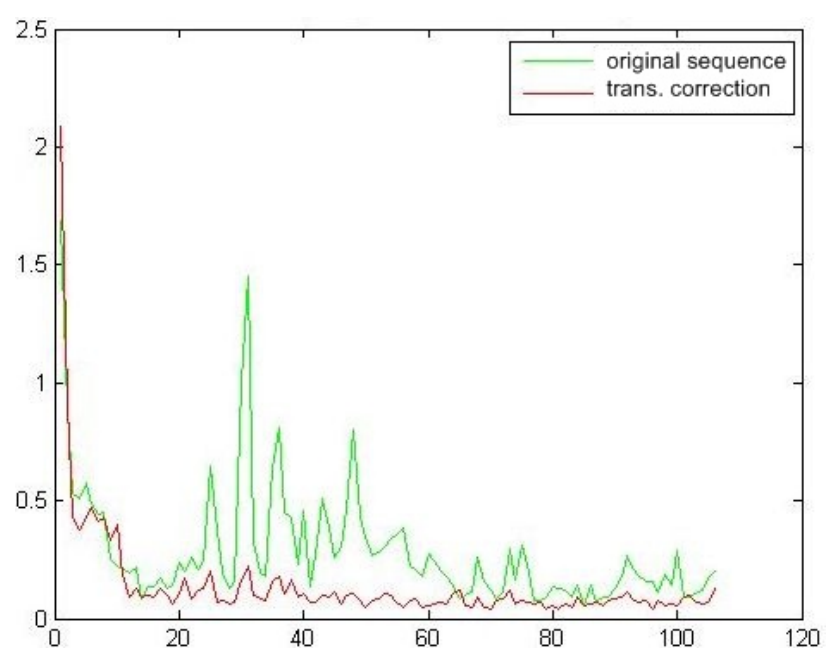

(b) Frequency Spectrum

Figure 3: An example of the mean dynamic for the cortex of one kidney for one data set is visualized in (a). Visually, the improvement of the perfusion assessment resulting in a smoother curve can be noticed. In (b) we quantitatively confirm this fact by presenting the mean frequency spectrum for the dynamics of the cortex. It can clearly be seen that the high frequency components of the dynamics, which are induced by patient movement, are successfully removed.

where $n_{\epsilon}$ denotes a differentiable approximation to the normalized gradient

$$
\mathbf{n}_{\epsilon}(I, \mathbf{x})=\frac{\nabla I(\mathbf{x})}{\|\nabla I(\mathbf{x})\|_{\epsilon}}, \text { with }\|\mathbf{x}\|_{\epsilon}=\sqrt{\mathbf{x}^{\top} \mathbf{x}+\epsilon} .
$$

For more details on NGF, please refer for example to Haber and Modersitzki. ${ }^{7}$ Since this measure employs normalized gradients, it is largely contrast-invariant. We evaluate the similarity measure only in the kidney region in order to discard regions of the image, which are irrelevant for the quantitative study. The image is automatically split vertically between the two segmented kidneys, such that the left and right kidney are treated independently.

In order to cope with the large motion and especially the drift of the mean motion over the time series, we perform the registration of all images to one reference image, instead of registering neighboring images to each other, since this would lead to error accumulation. Although registering neighboring images makes the intensity variation between images to be registered smaller, the error accumulation still leads to inferior results for the accurate estimation of the kinetic parameters.

The additional user interaction needed for our method consists of a selection of a reference slice in the DCE-MRI time series and a coarse manual segmentation of the kidney in this slice which can easily be performed by a large-size brush tool. Since the estimation of the kinetic parameters already requires user interaction, our motion correction method can easily be integrated in the clinical workflow without a significant overhead.

\subsection{Tracer Kinetic Modeling}

The dynamics of the contrast agent are analyzed using a 4-parameter two-compartment model (Figure 2). In general terms, the model visualized in Figure 2 is a separable two-compartment model with a single inlet in the central compartment. ${ }^{3}$ It provides a simplified description of the filtration processes that take place in the kidney cortex. The contrast agent is carried by a plasma flow $\left(F_{\mathrm{P}}\right)$ from an arterial region into the tissue. It first enters the tissue plasma, where it distributes over the plasma volume $\left(V_{\mathrm{P}}\right)$. A fraction of the entering contrast agent is filtered out of the vascular compartment and is carried by a tubular flow $\left(F_{\mathrm{T}}\right)$ into the tubular system where it distributes over the tubular volume $\left(V_{\mathrm{T}}\right)$. The contrast agent leaves the tissue carried by the outflow out of the vascular and tubular compartments.

The model can be solved by first solving the plasma compartment, then using the output out of the plasma compartment as an input to the tubular compartment. Using the fact that the residue function of a compartment is mono-exponential, the 


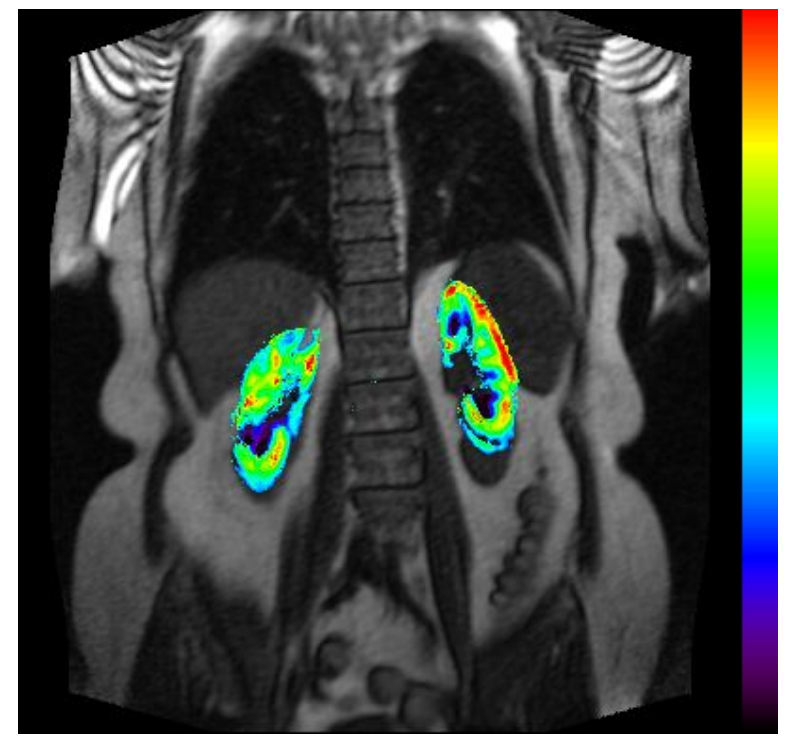

(a) Plasma Volume: original sequence

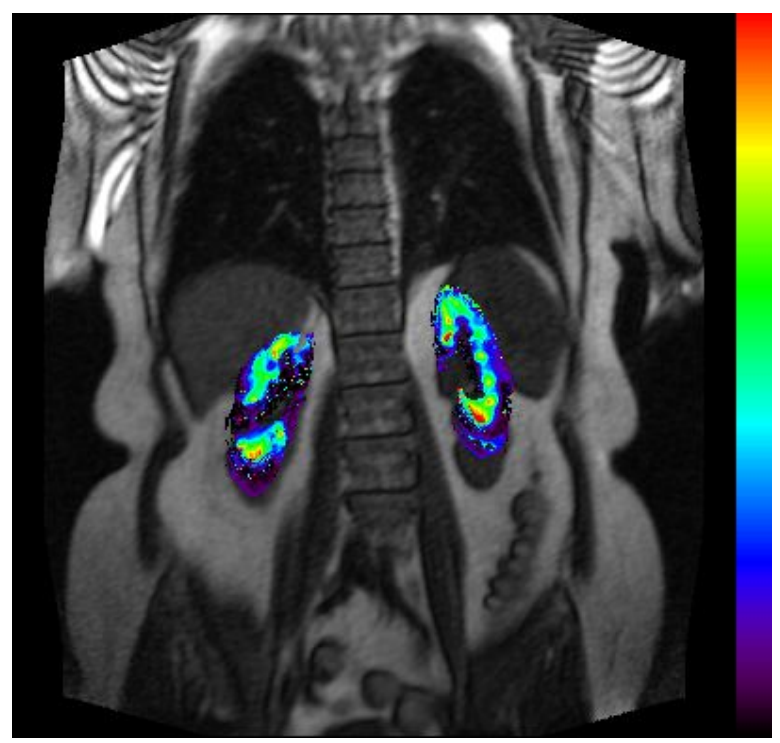

(c) Tubular Flow: original sequence

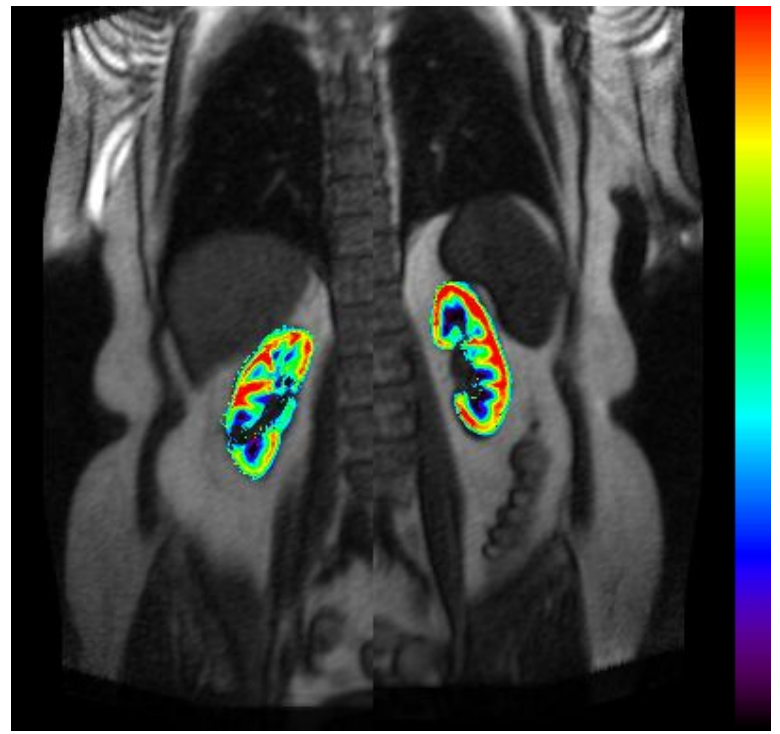

(b) Plasma Volume: after correction

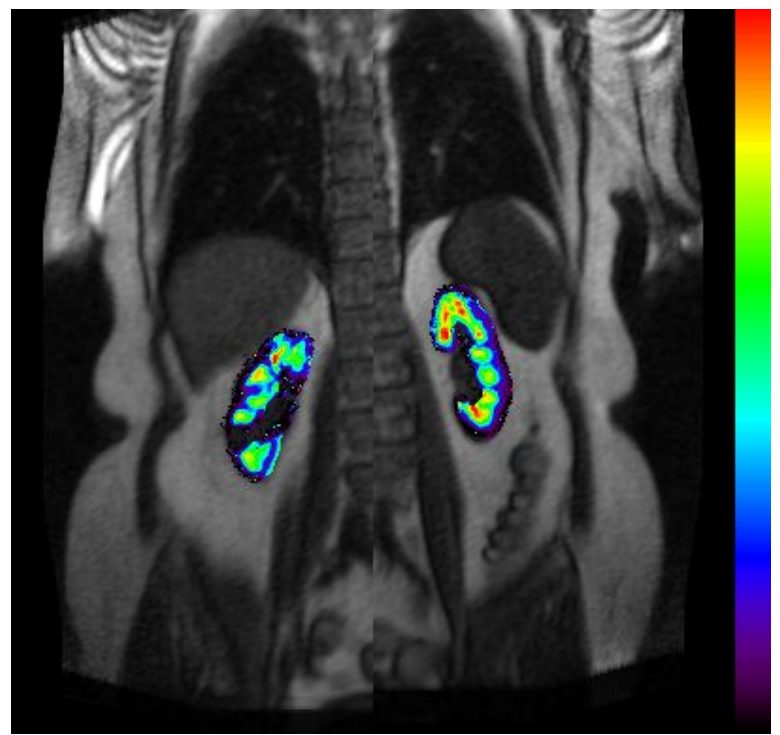

(d) Tubular Flow: after correction

Figure 4: An example for the results of the quantitative analysis before and after alignment correction is presented. In the left column the results using the original image sequence are presented: (a) Plasma Volume, (c) Tubular Flow. In the right column the respective results after translational alignment are presented. The results obtained after alignment have physically more meaningful values and more closely represent the behavior observed in the image series. (For a color-version, please see the electronic version of this paper.)

tissue concentration of tracer particles $C(t)$ (mmol tracer/ml tissue) in the two-compartment system is given by:

$$
C(t)=e^{-t / T_{\mathrm{P}}} * F_{\mathrm{P}} C_{\mathrm{A}}(t)+\left(F_{\mathrm{T}} / T_{\mathrm{P}}\right) e^{-t / T_{\mathrm{T}}} * e^{-t / T_{\mathrm{P}}} * C_{\mathrm{A}}(t)
$$

Here $*$ denotes convolution, the arterial input function $C_{\mathrm{A}}(t)(\mathrm{mmol}$ tracer $/ \mathrm{ml}$ plasma) is the concentration of contrast agent in the arterial plasma, $T_{\mathrm{P}}$ is the mean transit time of the contrast agent in the plasma, and $T_{\mathrm{T}}$ is the mean transit time of the contrast agent in the tubuli. 


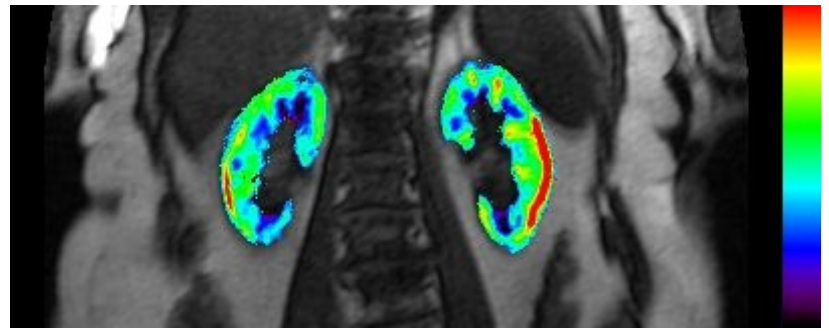

(a) Plasma Volume: original sequence

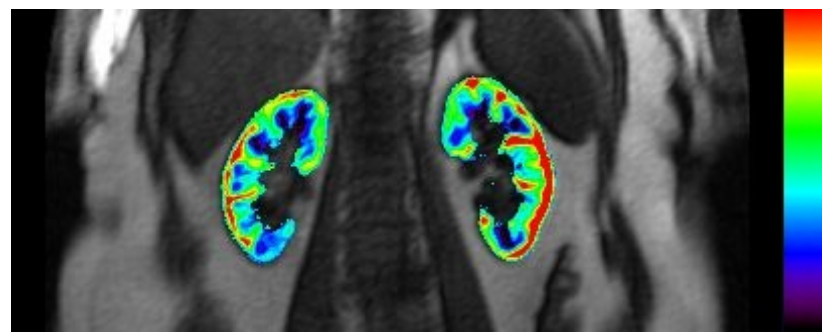

(c) Plasma Volume: after translational correction

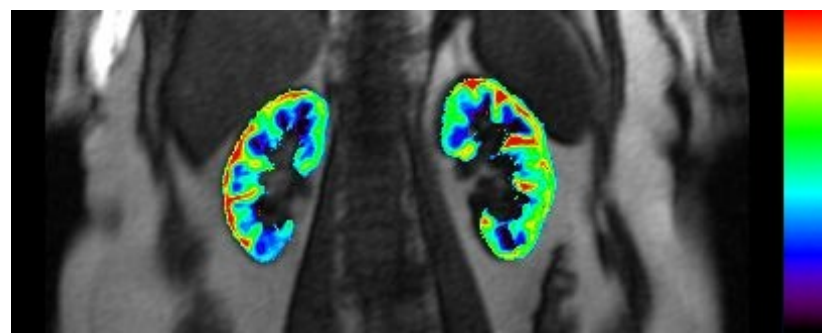

(e) Plasma Volume: after deformable correction

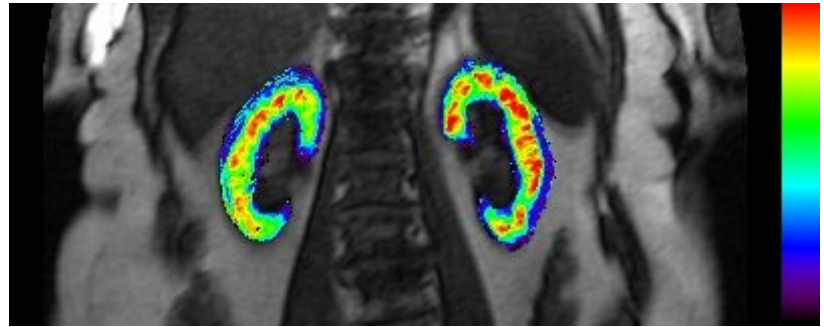

(b) Tubular Flow: original sequence

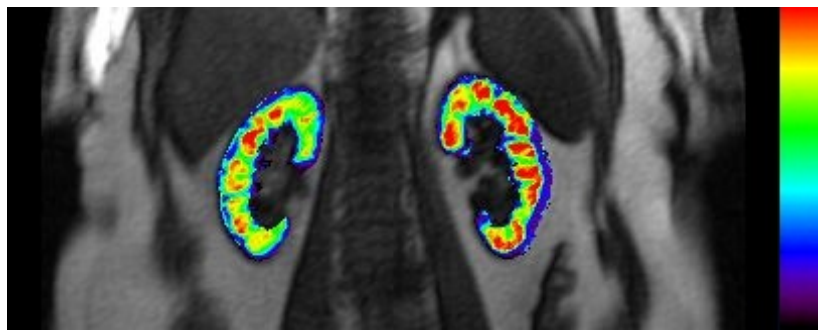

(d) Tubular Flow: after translational correction

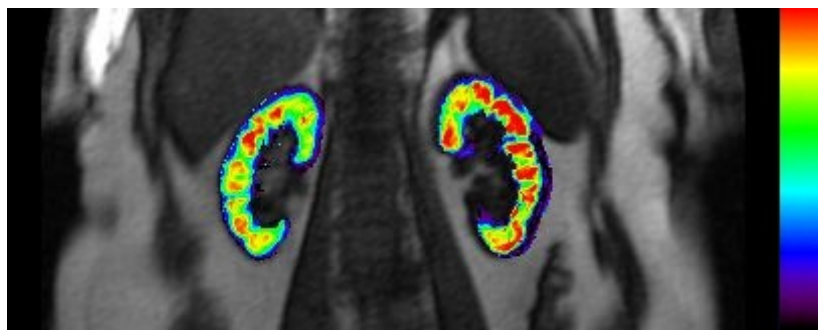

(f) Tubular Flow: after deformable correction

Figure 5: Example for the estimate of the plasma volume and tubular flow parameters: without correction (top row), with translational alignment (middle row), and with deformable registration (bottom row). Left column displays the plasma volume results while the right column displays the tubular flow results. Note that some unrealistic artifacts are introduced by deformable registration, for example in the left kidney (right-hand side in the image) for the plasma volume parameter. (For a color-version, please see the electronic version of this paper.)

\subsection{Estimating the Perfusion and Filtration Parameters}

A region of interest is drawn manually in the aorta, and the arterial input function is derived by averaging all pixels in this region for each dynamic. Contrast concentrations are approximated by signal enhancement, that is by subtracting the baseline from the dynamic signal. Arterial plasma concentrations are derived using a fixed hematocrit value of 0.45 . The two-compartment model is then fitted on a pixel-by-pixel basis for all pixels in the middle coronal slice. The analysis is first applied to the unregistered data, then repeated for the registered data. The fitted model parameters for all pixels within the kidney are color-coded and superimposed on the unenhanced baseline image for anatomical reference.

\section{RESULTS AND EVALUATION}

In this section, we briefly describe the data acquisition process and present the performed evaluation of the method.

\subsection{Data Acquisition}

We evaluate our method on 10 data sets of healthy volunteers as well as diseased patients, with time series containing 180-230 images. DCE-MRI was performed using a 1.5T and a 3T scanner. Four slices (3 coronal, 1 axial) are measured at $276 \mathrm{~ms}$ per slice, leading to a temporal resolution of $1.1 \mathrm{~s}$. 


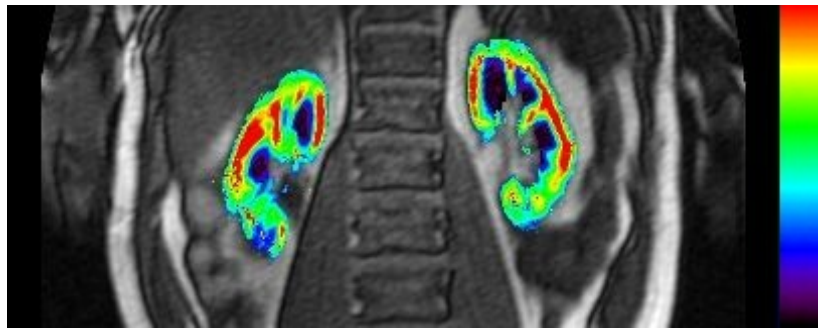

(a) Plasma Volume: original sequence

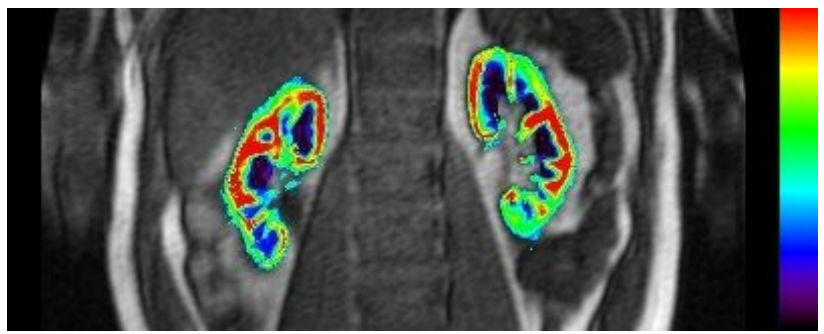

(c) Plasma Volume: after translational correction

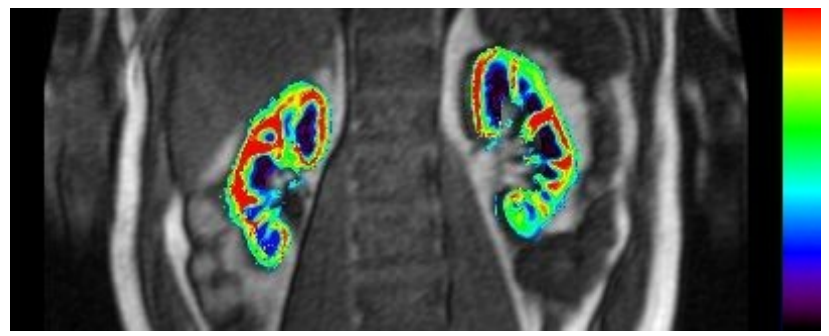

(e) Plasma Volume: after deformable correction

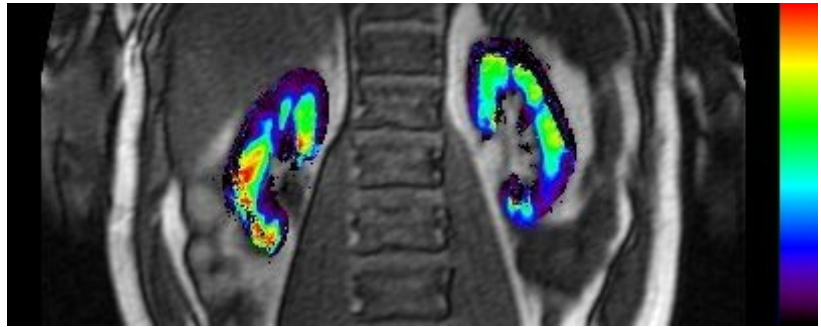

(b) Tubular Flow: original sequence

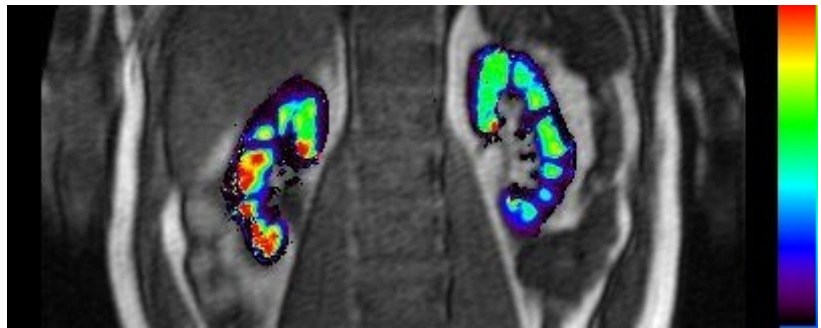

(d) Tubular Flow: after translational correction

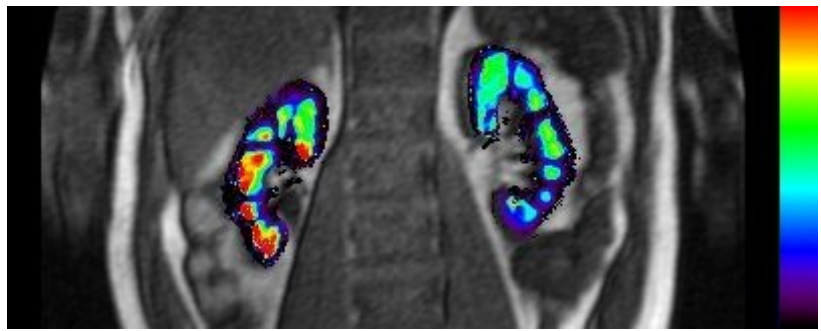

(f) Tubular Flow: after deformable correction

Figure 6: Another example for different results obtained when estimating the plasma volume and tubular flow parameters without correction (top row), with translational alignment (middle row), and with deformable registration (bottom row). Left column displays the plasma volume results while the right column displays the tubular flow results. (For a colorversion, please see the electronic version of this paper.)

\subsection{Method Evaluation}

The evaluation of the estimated kinetic parameters is a difficult issue, since the ground truth information is not directly available. Thus, the results mostly have to be evaluated by trained physicians, which are judging how plausible the estimated information appears.

In order to evaluate the performance of our method, we perform two tests. First, the dynamics are evaluated after motion correction and compared to the original dynamics. The corrected dynamics have a smoother shape and lack the breathing-induced jitter in the signal. We confirm this visual result by analyzing the frequency spectrum of the signal. For an example please see Figure 3. Furthermore, in order to evaluate the benefit of the method for the application of quantitative analysis of kinetic parameters, the perfusion and filtration parameters are estimated on the original input data and the same series after motion correction. We observe that the results after the performed motion correction are improved compared to the results computed on the original data sets. In all of the data sets, physically meaningless artifacts which are due to the kidney motion are successfully removed, without introducing any negative side-effects. Please compare Figures 4, 5 and 6 for a visualization of the estimated results on one of the data sets.

We also performed tests in which we compare the results of our method (translational correction) to the results obtained after a deformable registration using the same similarity measure is performed on the data. For deformable registration, we use a variational approach with normalized gradient fields (NGF) as similarity constraint. As initialization we perform the 
translational motion correction method. In spite of an extensive adjustment of the parameters for the deformable method, it was not possible to achieve deformable results, which were free of errors induced by the strong intensity variations on any of the data sets. These errors result in a wrong deformation of the kidney and this leads to wrong values for the kinetic parameters. For visualization of this behaviour, please compare Figures 5 and 6.

\section{CONCLUSION}

In this paper we present an approach for improvement of quantitative tracer kinetic studies by a template-matching based motion correction method. The step of motion correction is needed since otherwise unwanted artifacts are present in the parameter estimation, which are caused by the breathing motion of the patient. Thus, this step is crucial for automatic estimation of the tracer kinetic parameters.

For our template-matching method we use a contrast-invariant similarity measure and in order to obtain robust results and reduce the computation time, we constrain the motion to a translational model.

The evaluation on real data shows that the method is capable of improving the quantitative studies by removing the effects of the breathing-induced patient motion. The comparison to a deformable model with a large number of degrees of freedom indicates that the implicit robustness of the simpler translation motion model can lead to superior results.

\section{REFERENCES}

1. N. Grenier, O. Hauger, A. Cimpean, and V. Pérot, "Update of renal imaging," Seminars in Nuclear Medicine 36(1), pp. 3-15, 2006.

2. H. J. Michaely, S. Sourbron, O. Dietrich, U. Attenberger, M. F. Reiser, and S. O. Schoenberg, "Functional renal mr imaging: an overview," Abdom. Imaging , 2006.

3. J. A. Jacquez, Compartmental analysis in biology and medicine, University of Michigan Press Ann Arbor, 2nd ed., 1985.

4. R. Bansal and G. Funka-Lea, "Integrated image registration for cardiac mr perfusion data," Medical Image Computing and Computer-Assisted Intervention 2488, pp. 659-66, 2002.

5. Y. Sun, M.-P. Jolly, and J. M. Moura, "Contrast-invariant registration of cardiac and renal mr perfusion images," Medical Image Computing and Computer-Assisted Intervention 3216, pp. 903-910, 2004.

6. A. El-Baz, R. Fahmi, S. Yuksel, A. A. Farag, and W. Miller, "A new cad system for the evaluation of kidney diseases using dce-mri," Medical Image Computing and Computer-Assisted Intervention 4191, pp. 446-453, 2006.

7. E. Haber and J. Modersitzki, "Intensity gradient based registration and fusion of multi-modal images," Medical Image Computing and Computer-Assisted Intervention 4191, pp. 726-733, 2006. 